\title{
BRAINSTEM DEATH AND ORGAN DONATION
}

\author{
L.D.Walallawita* \\ Consultant Anaesthetist, BH Avissawella. \\ *Corresponding author: lekha_w@hotmail.com
}

Key Words: Brainstem Death, Organ Transplantation

The one organ which we cannot substitute or replace is the brain. Death of a person is now defined in terms of death of the brainstem. When brainstem death was formally defined by the Conference of Medical Royal Colleges and their Faculties in the UK in 1976, a concept as well as a description of a pathological process was introduced into medical practice. This article deals with the pathophysiology of brain injury, definition of Brain Stem Death (BSD), process of BSD testing, , history of organ transplantation and briefly explores the care of the organ donor, the Sri Lankan perspective and the role of the anaesthesiologist.

\section{Pathophysiology of brain injury}

The brain is susceptible to permanent damage for a number of reasons, key factors being high Oxygen $\left(\mathrm{O}_{2}\right)$ demand, deleterious impact of swelling within a fixed volume, specific anatomical considerations and the inability of neural tissues to undergo repair.

The cerebral metabolic rate for oxygen $(3.5 \mathrm{ml} / 100 \mathrm{~g} / \mathrm{min})$ accounts for around $20 \%$ of the total resting $\mathrm{O}_{2}$ consumption of the body, with cerebral blood flow comprising almost $15 \%$ of the cardiac output $(750 \mathrm{ml} / \mathrm{min})$. There is very little reserve, therefore, hypoxia or ischaemia and unconsciousness occurs within 10 seconds of interruption of the cerebral blood supply.

Susceptibility to hypoxic injury is not uniform throughout the brain, the cerebral cortex being most vulnerable, followed by the cerebral nuclei. The cardiorespiratory centres within the brainstem are relatively resistant to hypoxic injury; this explains the persistent vegetative state
(PVS) as an end product of severe injury characterized by cortical death and lack of awareness, but intact ventilatory drive.

Although the primary purpose of the skull is to protect the underlying brain, once injury has occurred, the constraints of a rigid container on brain swelling cause an increase in Intra Cranial Pressure (ICP), thereby compromising cerebral oxygen delivery. The subsequent ischaemia further contributes to brain oedema triggering a progressive spiral of rising pressure and aggravated ischaemia. The ultimate effect of a globally high ICP is death of the brainstem due to coning (i.e. downwards extrusion through the foramen magnum).

\section{Definition and Diagnosis of BSD}

According to the brain death criteria proposed by the Conference of Medical Royal Colleges of UK in 1976 and 1979, brainstem death is regarded as "irreversible loss of the capacity for consciousness, combined with irreversible loss of the capacity to breathe." On this basis, BSD is considered to equate with generic death of the individual. BSD testing is subject to strict control since diagnosis of BSD is now closely linked with the harvesting of organs for transplantation. However, in the UK, clinical testing alone by two medical practitioners holding full GMC registration for more than 5 years, one of whom should be a Consultant, is sufficient for diagnosis of BSD as long as they are competent to undertake the tests, experienced in interpreting the results independent of the transplant team. Confirmatory tests such as 4 vessel angiography, Trans Cranial Dopplar and radio-isotope scanning 
have been used occasionally in the UK when clinical testing has not been possible ( eg. when hypoxia precludes apnoea testing, local cranial nerve lesions, presence of long acting anaesthetic/sedative agents). In the majority of cases without any of these confounding factors, confirmatory factors have not been shown to increase the accuracy of the clinical diagnosis of BSD.

\section{The process of BSD testing}

Before a diagnosis of BSD can be made, certain essential preconditions must be met and it is ensured that none of the exclusion criteria are present.

\section{Preconditions}

1. Patient should be deeply unconscious, apnoeic and maintained on a ventilator.

2. Cause of the coma must be irreversible brain damage of known aetiology.

Exclusion Criteria

1. Hypothermia $\left(<35^{\circ} \mathrm{c}\right)$

2. Drugs (cerebral depressants, muscle relaxants etc.)

3. Acid-base abnormality

4. Potentially reversible metabolic, endocrine or circulatory abnormality (uncontrolled Diabetes Mellitus (DM), uraemia, hyponatraemia, Addison's, disease hepatic encephalopathy, thyrotoxicosis)

5. Severe hypotension, markedly elevated arterial carbondioxide tension $\left(\mathrm{PaCO}_{2}\right)$

\section{Clinical Testing of Brainstem Function}

These bedside tests are designed to test function of cranial nerve nuclei and vital centres of the brainstem. Testing is considered when there is no prospect of recovery in a patient otherwise stable in terms of organ system function. Testing is undertaken by 2 doctors who have been registered in the respective medical council for more than 5 years, are experienced in the diagnosis of BSD and are not members of the transplant team. At least one should be a Consultant. They may carry out the tests together but always two sets of tests should be performed to avoid observer error.

Timing of the first set of tests will be influenced by the underlying condition. For example, the irreversible nature of the damage after a head injury may be more apparent than after a cardiac arrest .In any case, it is recommended that at least 6 hours be allowed to lapse after the insult to allow time for any improvement but after cardiac arrest, it is recommended that at least 24 hours elapse before testing.

Two sets of tests are performed to remove the risk of observer error. There is no statutory interval between tests but interval should be sufficient to reassure all parties involved.

Death should not be pronounced until the second set of tests are completed but the legal time of death is the time of completion of the first set of tests. After the second set of tests ventilation can be discontinued or the patient can be taken for organ donation. The coroner needs to be informed in the usual way if the BSD is not clearly a result of natural causes.

\section{Brainstem testing}

Brainstem death tests are designed to test function of cranial nerve nuclei and vital centres in the brainstem. Documentation of all test results should be carried out on the patient's notes by the 2 doctors who performed the clinical testing. It is desirable if a template approved by the Ministry of Health is available for this purpose. The features of BSD are as follows:

i. The pupils are fixed with no direct or consensual response to light (CN II, III \& sympathetic outflow). It is not essential that the pupils be maximally dilated or equal in size and shape.

ii. There is no corneal reflex on stimulation, conventionally elicited with cotton wool to avoid abrasion (CN V and VII)

iii. There should be no motor response within the cranial nerve distribution in response to stimulation of any somatic area (CN V and VII). There should be no limb response to supra- orbital pressure (movements of limbs and trunk may occur due to spinal reflexes after BSD which, if not explained, may prove distressing to both relatives and staff).

iv. The vestibulo-ocular reflexes should be absent on caloric testing (VIII and III) 
The tympanic membrane should first be visualized and any wax or debris removed as necessary. The head is flexed to $30^{\circ}$ for this test and at least $50 \mathrm{ml}$ of ice-cold water is instilled over one minute deep into each external auditory meatus. The eyelids are held open to observe any eye movements, mainly nystagmus, during or after the instillation of water. If the performance of these tests is impossible on one side because of local injury or disease, the tests are not invalidated.

v. There should be no gag reflex (contraction of the soft palate and oropharynx) when the posterior pharyngeal wall is stimulated with the spatula (CN IX)

vi. There should be no cough or other reflex response to passage of a suction catheter down the endotracheal tube and stimulation of the carina $(\mathrm{CN} \mathrm{X})$

\section{Apnoea Testing}

No respiratory movements should occur when the patient is disconnected from the ventilator, with chest and abdomen exposed and directly observed. The patient should be pre-oxygenated and the $\mathrm{PaCO}_{2}$ allowed to rise to $5 \mathrm{kPa}$ prior to disconnection from the ventilator. Hypoxia should be prevented by $\mathrm{O}_{2}$ insufflation through a catheter within the ET tube. Sufficient time should be allowed for the $\mathrm{PaCO}_{2}$ to rise to 6.65 $\mathrm{kPa}$ to exceed the normal threshold for stimulation of respiration, confirmed by measurement of arterial blood gases.

\section{Importance of diagnosing BSD}

Diagnosing BSD is simply good intensive care practice; it allows discontinuation of intensive care treatment in a patient with no chance of recovery and allows the family to come to terms with the situation. Critical care facility, as we are all aware, is scarce and expensive and therefore should be put into maximum use. Timely diagnosis of BSD makes it possible for efficient utilization of intensive care resources. Moreover, diagnosing BSD allows consideration of potential organ donation. It is after all, one way of salvaging some good from personal disaster from the relatives' point of view and to some it would mean " life after death". Last but not least, diagnosing BSD using the clinical tests which has a sensitivity of $100 \%$ is doubly important in a developing country like ours, since it is a simple, inexpensive but highly robust method of establishing a diagnosis.

\section{History of Organ Transplanation}

The first documented organ transplant took place in France in 1906 when Jaboulay grafted a pig kidney into the ante-cubital fossa of a woman dying of renal failure. Voronoy in Russia performed the first human to human kidney transplant in 1933 using a donor kidney from a woman who had died 6 hours before. Unsurprisingly all of these procedures failed and it was only in the 1940's that the role of the immune system in rejection of the transplanted organs was recognized and over the next 2 decades work began on the immunosuppressive therapy which would make transplantation possible. Advent of artificial ventilation and intensive care in the 1950's resulted in the first brainstem dead heart-beating donors.

\section{Identification of the potential organ donor}

Donors should usually be $<75$ years of age with no medical or social risk factors for HIV, Hepatitis B or C infection. There must be no evidence of untreated sepsis or malignancy apart from tumours of the central nervous system

(CNS). However these contra-indications are often relative and much depends on recipient characteristics - for example an organ from a Hepatitis ' $C$ ' positive donor may be suitable for transplant if the proposed recipient is also Hepatitis 'C' positive.

\section{Approach to the donor's family}

The family should be informed of progress at every stage. They will usually be facing a sudden, catastrophic loss and a thoughtful and a compassionate approch is essential. It is important to discuss the meaning of BSD and its implications in appropriate detail. The concept of BSD may be difficult for many people to understand and this may require time and careful explanation.

\section{Situation in SriLanka}

Chronic Renal Failure is rampant among the farming communities in North Central Province, 
North Western Province, Uva Province and the Eastern Province. In Anuradhapura district alone, it is estimated that there are about 4000 patients in end stage renal failure. Every week about 15 20 new patients get registered in the clinic run by the renal unit. So much so, at present well over 8000 patients are awaiting kidney transplantation. Unfortunately only a few transplants are done per month - mostly from live related and live nonrelated donors and so far just 6 kidney transplants have been done from heart-beating cadaveric donors in Sri Lanka. This is in spite of many young people who are fit to donate, dying of head injury daily.

The Kidney Transplant Support Foundation in Sri Lanka has come forward to find a remedy for this most unfavourable scenario, bringing a ray of hope to thousands of innocent patients. In March 2009 they were able to launch a National Organ Donor Programme a web based database, which will enable registered organ donors across the country to altruistically donate a kidney, or agree to make their organs available for organ donation after death, paving way to save many lives in SriLanka.

\section{How can we help?}

Our role as critical care Specialists enable us to deal with extremely sensitive issues like BSD with compassion and efficiency. No one else would be in a better position to create an awareness among the general public about the concept of brainstem death and the plight of prospective recipients, thus promoting the" 'Live and Let Live" and Die and Let Live" concepts which encourage people to become active or inactive donors.

Majority of the ICU's across the country are run under the supervision of anaesthetists. Therefore, diagnosing BSD and looking after the heartbeating cadaveric donor till organ harvesting is carried-out, automatically becomes our responsibility. The distressed relatives who may naturally be undergoing a most trying time in their lives should be handled with utmost care and compassion, helping them to come to terms with the situation and guiding them to take the correct decisions.
As attending physicians who look after patients and finally make the diagnosis of BSD, we are precluded from becoming members of the transplant team. However, it is within our purview to facilitate the work of the transplant coordinator.

The general standards of intensive care treatment provided for the patient before BSD must continue after the diagnosis to maintain the organs in the optimum condition. At the same time, there are several aspects of management that are specific to the potential donor like "hormone resuscitation" which have been shown to increase the number of transplantable organs. Liaising with the administration to acquire logistic support for the purpose of management of the potential heart-beating organ donor will be another one of our responsibilities.
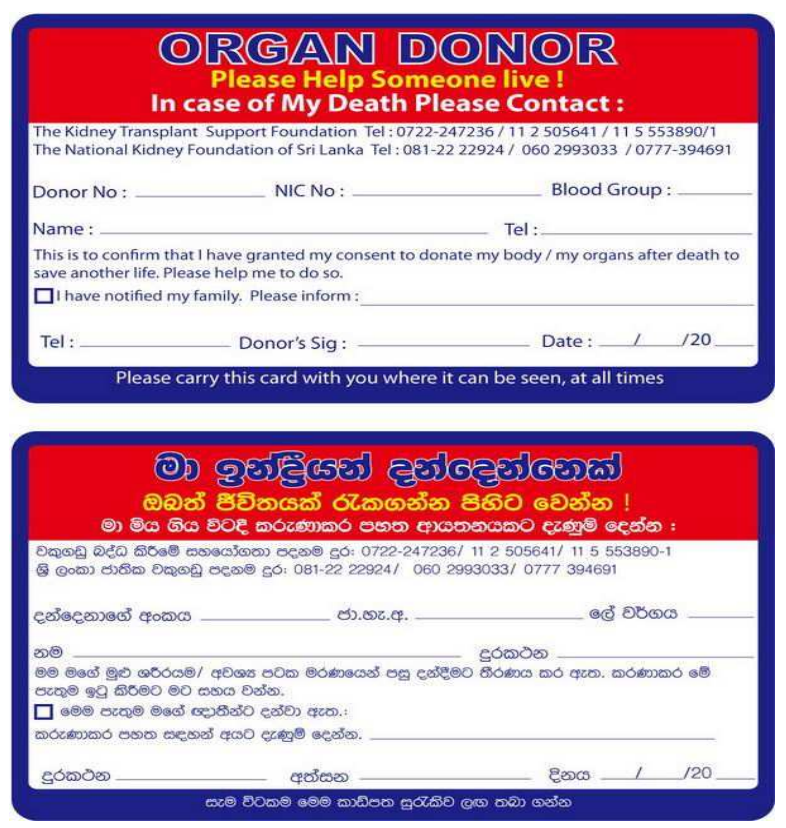

\section{References}

1. Conference of Medical Royal Colleges and their Faculties in the United Kingdom Diagnosis of brain death. BMJ 1976; ii:118 - 8

2. Department of Health. A Code of Practice for The Diagnosis of BSD. London HMSO, 1998

3. Donation of organs for transplantation / The management of the potential organ donor. Intensive Care Society Publications, June 1999 